Jul 1st, 12:00 AM

\title{
An agglomeration payment for cost-effective biodiversity conservation in spatially structured landscapes
}

Martin Drechsler

Frank Wätzold

Karin Johst

Jason F. Shogren

Follow this and additional works at: https://scholarsarchive.byu.edu/iemssconference

Drechsler, Martin; Wätzold, Frank; Johst, Karin; and Shogren, Jason F., "An agglomeration payment for cost-effective biodiversity conservation in spatially structured landscapes" (2010). International Congress on Environmental Modelling and Software. 49. https://scholarsarchive.byu.edu/iemssconference/2010/all/49

This Event is brought to you for free and open access by the Civil and Environmental Engineering at BYU ScholarsArchive. It has been accepted for inclusion in International Congress on Environmental Modelling and Software by an authorized administrator of BYU ScholarsArchive. For more information, please contact scholarsarchive@byu.edu, ellen_amatangelo@byu.edu. 


\title{
An agglomeration payment for cost-effective biodiversity conservation in spatially structured landscapes
}

\author{
Martin Drechsler ${ }^{\mathrm{a}}$, Frank Wätzold ${ }^{\mathrm{b}}$, Karin Johst ${ }^{\mathrm{c}}$, Jason F. Shogren ${ }^{\mathrm{d}}$ \\ ${ }^{a}$ Helmholtz-Centre for Environmental Research - UFZ, martin.drechsler@ufz.de \\ ${ }^{\mathrm{b}}$ Ernst Moritz Arndt University of Greifswald, frank.waetzold@ufz.de \\ ${ }^{\mathrm{c}}$ Helmholtz-Centre for Environmental Research - UFZ, karin.johst@ufz.de. \\ ${ }^{\mathrm{d}}$ University of Wyoming, JRamses@uwyo.edu
}

\begin{abstract}
Connected habitats are ecologically more valuable than isolated habitats for many species. A key challenge when designing payments for biodiversity in fragmented landscapes is to increase the spatial connectivity of habitats. Based on the idea of an agglomeration bonus we consider a scheme in which land-owners only receive payments if habitats are arranged in an ecologically favourable configuration. We compare the costeffectiveness of agglomeration payments to spatially homogeneous payments on a conceptual level. Our results suggest that positive efficiency gains exist for agglomeration payments. We use Large Blue butterfly habitat in Germany as a specific case study, and find the agglomeration payments may lead to cost-savings of nearly 70 percent relative to homogenous payments.
\end{abstract}

Keywords: agglomeration bonus; biodiversity conservation; cost-effectiveness, ecologicaleconomic modelling; spatial heterogeneity.

\section{INTRODUCTION}

Compensation schemes in which land owners receive payments for voluntarily managing their land in a biodiversity-enhancing manner have become one of the most important instruments for biodiversity conservation worldwide (cf. European Commission [2005], Landell-Mills and Porras [2002]).

These voluntary schemes are necessary because property rights are frequently allocated so landowners have considerable latitude to manage their land in their own private interest. Experience with the US Endangered Species Act also demonstrates that forcing owners of land with endangered species to carry out conservation measures might encourage them to eradicate these species to escape the burden of conservation costs (Brown and Shogren [1998]).

One key challenge when designing effective biodiversity conservation compensation schemes is to account for the spatial arrangement of habitats (e.g., Knight and Landres [1998], Raymond [2005]). The ecological literature addresses how specific habitats should be spatially allocated to maximise the targeted ecological benefit, such as population size, species viability, and so on (see, e.g, Frank and Wissel [2002]). In spatially structured landscapes, species populations exist as so-called metapopulations, which consist of subpopulations each of which inhabits a habitat patch. If individual members of the species can move between patches, the subpopulations interact. In general, this exchange of individuals is beneficial for the survival of the metapopulation, resulting in the general rule for given total habitat area connected habitats are ecologically more valuable than isolated habitats (e.g., Hanski [1999]).

Given the voluntary nature of payment schemes, the question is how to induce land-owners to select land for conservation so that habitats are connected? Many payments are spatially homogeneous - every land-owner receives the same payment for a particular conservation measure. Such homogeneous payment schemes generate an ecologically valuable spatial 
configuration only if it contains the least costly patches. In contrast, Parkhurst et al. [2002] suggested an alternative scheme - the 'agglomeration bonus.' The agglomeration bonus provides an incentive to land-owners to generate a valuable configuration. The idea of an agglomeration bonus is a premium - a bonus - is paid on top of a standard payment for managing land in a biodiversity-enhancing manner if the managed patches are arranged in a specific spatial configuration. This requires cooperation among land owners and research about the agglomeration bonus has so far focussed on the cooperation problem (e.g. Parkhurst et al. [2002], Parkhurst and Shogren [2007], [2008]).

Herein we bypass the cooperation problem and explore the cost-effectiveness question: does an agglomeration bonus lead to a higher ecological output for a given budget than homogeneous payments? This question raises the concern that the agglomeration bonus leads to higher ecological benefits but it can also lead to higher costs. It may be necessary to include costly patches to achieve a desired spatial configuration. This suggests a tradeoff exists between maximising total habitat size with inferior spatial configuration and optimising spatial configuration with less habitat size.

This paper addresses this question - we compare the cost-effectiveness of an agglomeration bonus against a spatially homogeneous payment. We compare two 'pure' payment schemes: (i) payments are only made when an ecologically beneficial spatial configuration is generated (agglomeration payment), and (ii) payments are made independent of habitat location (spatially homogeneous payment). We first explore a conceptual analysis on a fictitious landscape to gain insight into how economic and ecological parameters affect the cost-effectiveness of an agglomeration payment. We then compare the cost-effectiveness of homogeneous and agglomeration payments for a real conservation problem: the protection of an endangered butterfly species (Large Blue, Maculinea teleius) in a region around the city of Landau, Germany.

\section{INTRODUCTION OF AGGLOMERATION PAYMENTS}

Consider a landscape with $N$ discrete habitat patches. These may be regarded as islands within a matrix of uninhabitable habitat (cf. Fig. 1) but may also share their boundaries (e.g. a regular or irregular grid with each cell representing a patch). Let $\mathbf{r}_{\mathbf{i}}=\left(x_{i}, y_{i}\right)$ denote the spatial location and $a_{i}$ the size of patch $i(i=1 \ldots N)$ and $z_{i}$ the land-owner's decision to manage patch $i$ in a species-friendly "green" manner $\left(z_{i}=1\right)$ or conventional manner $\left(z_{i}=0\right)$. The vector $\mathbf{z}=\left(z_{1}, \ldots, z_{N}\right)$ then characterises the spatial arrangement of green patches. The opportunity cost (per habitat patch) to manage patch $i$ in a green manner is denoted as $c_{i}$. For convenience, assume each land-owner possesses one patch so the words 'patch' and 'land-owner' can be used synonymously.

Without a detailed specification, we assume an ecological benefit function $\Gamma\left(a_{i}, \mathbf{r}_{i}, z_{i}\right.$; $i=1 \ldots N)$ that increases if the distances between the patches, determined by their locations $\mathbf{r}_{i}$, decrease. Rather than considering complicated frequency distributions of these pairwise distances, we focus on a simple surrogate variable, habitat density, which (for given number of habitat patches) is inversely related to the patch distances. We consider the ecological benefit $\Gamma$ can be larger by increasing the density of green patches, $\rho$, in some part of the landscape, denoted as $R$. The density of green patches in landscape part $R$ is defined as the total area of green patches contained in $R$ divided by the total area $(|R|)$ of $R$ :

For simplicity we assume that $\mathrm{R}$ has a rectangular shape. It can be of any size and have any location in the landscape. Patches outside the rectangle do not receive any payment and are managed conventionally. In mathematical terms, a payment $p$ is paid to land-owner $i$ if and only if

$$
z_{i}=1 \text { and } r_{i} \in R \text { and } \rho(R)>\rho_{\min }
$$

A payment scheme is defined by the payment $p$ and the threshold density $\rho_{\min }$. Note for purpose of simplification we assume there is only a payment if the conditions of Eq. (2) are met, i.e., no other, space-independent payment is considered. We assume that the agency 
only knows the mean and variance of the costs $\left(m_{c}\right.$ and $\left.\sigma_{c}\right)$ but has no information about the individual conservation $\operatorname{costs} c_{i}$.

For the response of the land-owners to the payment scheme $\left(p, \rho_{\min }\right)$ we assume that landowner $i$ only participates in the scheme if the profit from participation is positive $\left(\pi_{i}>0\right)$ with $\pi_{i}$ being determined by

$$
\pi_{i}=p-c_{i}
$$

Side-payments $s_{i}$ arise if the individual profit of land-owner $i$ from scheme participation is negative but her contribution is required to generate a desired rectangle. In this case, $s_{i}>0$ means land-owner $i$ receives side payments and $s_{i}<0$ means she offers them. We assume that land-owners know other land-owners' conservation costs $c_{i}$ and cooperate. The level of the side payment is determined through some kind of Coasian bargaining that renders the profits of both parties positive. This implies that land-owners select a rectangle if the aggregated profit from all land-owners, i.e. the sum over all $\pi_{i}$, is positive. They eventually decide so the total profit from participation of all land-owners in the landscape,

$$
\Pi=\sum_{i=1}^{N} z_{i} a_{i}\left(p-c_{i}+s_{i}\right)=\sum_{i=1}^{N} z_{i} a_{i}\left(p-c_{i}\right)
$$

is maximised. The side payments drop out in Eq. (4) as the sum over all side payments is zero. Having agreed upon a rectangle, the land-owners inform the agency about its location and size as well as the locations of the green patches. The resulting budget that has to be spent is

$$
B=\sum_{i=1}^{N} z_{i} a_{i} p
$$

The spatial configuration of green patches that maximises $\Gamma$ and represents the farmers' choice is determined by forming all possible distinctive rectangles in the landscape (two rectangles are distinctive if they contain a different subset of patches). In each rectangle, profit is maximised under the constraint Eq. (2). If the threshold density is too high or $p$ too small compared to $c_{i}$, a positive profit may be unachievable. Here the maximum profit is zero and is achieved through $z_{i}=0$ for all $i$ and the corresponding ecological benefit is zero.

\section{FUNCTIONING OF THE AGGLOMERATION PAYMENT}

\subsection{Illustration of basic principle}

To demonstrate the idea behind the agglomeration payment, consider now the artificial landscape in Fig.1, in which all patches have equal size $a$ and are arranged on a regular grid. The numbers in the circles represent the $\operatorname{costs} c_{i}$. With a payment of $p=2.1$ and a density threshold $\rho_{\min }=0$ (representing homogenous compensation payments) the three patches with $c=2$ are turned into green patches for a budget of 6.3. These three patches, however, are distant from each other and it would be desirable ecologically to have them closer together. This is achieved, e.g., by offering a payment of $p=3.1$ under the constraint that a green patch density of $\rho_{\min }=0.5 a / d^{2}$ is exceeded, where $d$ represents the distance between two adjacent grid points.

A number of possible rectangles $R$ can be formed that fulfil Eq. (4). The one that maximises the land-owners' total profit is in the lower right corner with $\Pi=3 p-2-2-3=2.3$ (Eq. 4). The required budget (Eq. 5) for these three green patches is $B=3 p=9.3$. It is higher than the budget required for $p=2.1$ without density threshold, because not the three least expensive patches are selected. We call this budget-increasing impact the patch restriction effect. In contrast, the connectivity between the patches, and the ecological effectiveness, has increased - an outcome we call the connectivity effect. Since the two effects point into 
opposite directions, it is unclear a priori whether the agglomeration payment is more costeffective than homogenous payments. In the next section we investigate this question in a comprehensive manner on a general model.

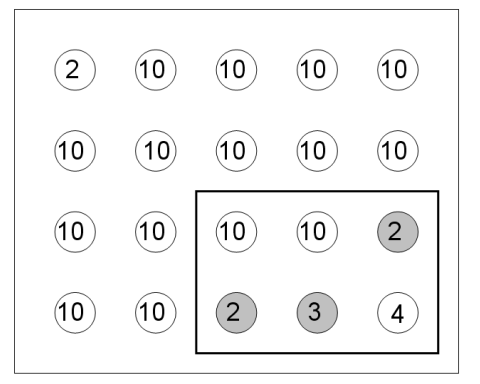

Figure 1: Artificial landscape of patches of identical size with different costs (given by the numbers). Patches filled grey are "green" patches managed as habitat. For further details, see text.

\subsection{Description of a conservation problem in a fictitious landscape}

We now consider the conservation problem in a fictitious landscape which we use to illustrate the cost-effectiveness of an agglomeration payment in a general manner. We use a general function to measure the connectivity of green habitat patches:

$$
\Gamma=\sum_{i=1}^{N} z_{i} \sum_{j=i+1}^{N} z_{j} \exp \left(-\alpha d_{i j}\right) \quad \text { with } \quad d_{i j}=\sqrt{\left(x_{i}-x_{j}\right)^{2}+\left(y_{i}-y_{j}\right)^{2}}
$$

where $d_{i j}$ is the distance between two patches $i$ and $j$ witrh coordinates $\left(x_{i}, y_{i}\right)$ and $\left(x_{j}, y_{j}\right)$, respectively. Equation (6) is based on a standard ecological model for the dispersal of individuals from one habitat patch to another (e.g, Hanski [1999]). Parameter $\alpha$ is the inverse of the mean species dispersal range. According to the theory of metapopulations (e.g., Hanski [1999]), $\Gamma$ is a good predictor for the survival probability of a metapopulation inhabiting the landscape.

With this measure of ecological benefit, an agglomeration payment scheme [defined as a combination of payment and density threshold $\left.\left(p, \rho_{\min }\right)\right]$ is more cost-effective than another agglomeration payment scheme if it leads to higher $\Gamma$ for a given budget. For the general analysis we consider a landscape where the patches are arranged on a regular square grid with length and width given by $d N^{1 / 2}$. Here $N$ is the total number of patches and $d$ is the distance between two adjacent grid points. The costs of patches $c_{i}$ are drawn randomly from a uniform distribution with mean $m_{c}$ and width $2 \sigma_{c}$. Costs are assumed to be spatially uncorrelated (we discuss this assumption in Section 5).

We consider a landscape of 100 patches, and we assume two levels of the dispersal parameter $\alpha$ : $\alpha_{1}=1 / d$ for a species with short-range dispersal and $\alpha_{2}=1 /\left(d N^{1 / 2}\right)$ for a species that can reach any patch in the landscape with high probability.

\subsection{Analysis of the demonstrational conservation problem}

We now define a landscape by the number of patches $(N)$, the distance between grid points $(d)$, patch size $(a)$, and mean and variation of costs $\left(m_{c}\right.$ and $\left.\sigma_{c}\right)$. Since the costs $c_{i}$ are uncertain, we randomly sample them for all patches 100 times. Each of the 100 random sets of costs $\left\{c_{i}\right\}_{i=1 \ldots N}$ may be denoted as a "cost landscape". For each cost landscape $\left\{c_{i}\right\}$ we examine 200 payment schemes $\left(p, \rho_{\min }\right)$. Means and standard deviations of the resulting connectivity $\Gamma$, the corresponding budget and the land-owners' total profit are calculated for each payment scheme over the 100 random cost landscapes. 
Some of the model parameters have a trivial effect on the results. Without loss of generality we set $m_{c}=d=a=1$ and consider three scenarios: (a) $\sigma_{c}=0.1, \alpha=1 / d$, (b) $\sigma_{c}=0.1, \alpha=1 /\left(N^{1 / 2}\right)$, (c) $\sigma_{c}=0.5, \alpha=1 / d$. In scenario a, the cost variation is small ( $10 \%$ of the mean) and the species dispersal range $(1 / \alpha)$ is small and equals the distance $d$ between neighboured patches. In scenario $\mathrm{b}$ the species dispersal range is increased to about the diameter $\left(d N^{1 / 2}\right)$ of the landscape; in scenario $\mathrm{c}$ the cost variation is increased to $50 \%$ of the mean.

\subsection{Results}

Figures 2a-c show the results for the three scenarios. All figures show the connectivity $\Gamma$ as a function of the budget for various density thresholds $\rho_{\min }$. The density threshold $\rho_{\min }=0$ (solid line in Fig. 2) represents homogeneous payments; the expected cost-effectiveness improvements of the agglomeration payment are presented in Fig. 2 for various $\rho_{\min }$.
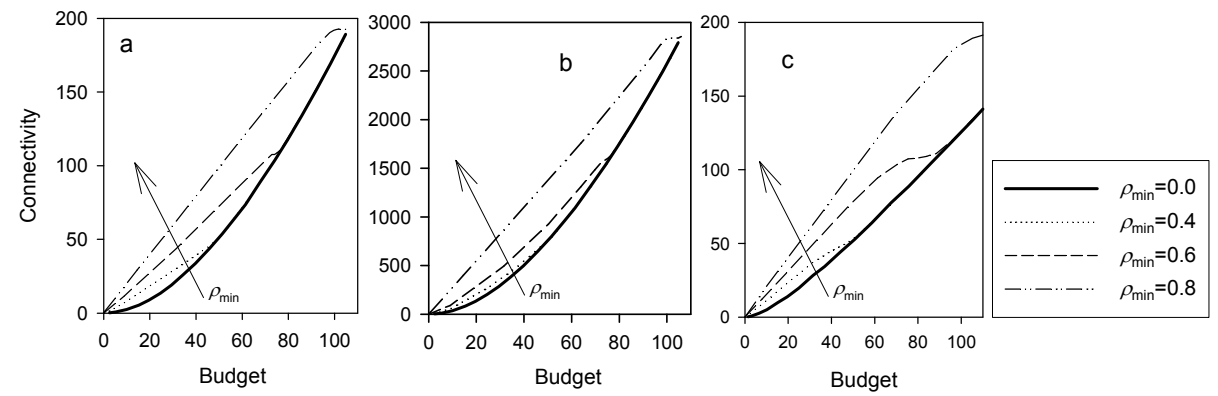

Figure 2: Mean connectivity versus mean budget (measured in units of mean patch cost $m_{c}$ ) for the different levels of $\rho_{\min }$. Solid bold line: $\rho_{\min }=0$ (equivalent to homogeneous payments). Dashed lines: $\rho_{\min }$ increasing from bottom to top with levels $0.2,0.4,0.6,0.8$. Panels (a)-(c) represent scenarios (a)-(c). Budget and connectivity are averages over the 100 random cost landscapes $\left\{c_{i}\right\}$.

In all scenarios of Fig. 2 the cost-effectiveness of a payment scheme increases with $\rho_{\min }$, so all agglomeration payment schemes with $\rho_{\min }>0$ lead to efficiency gains compared to homogenous payments. Why is this so, given the patch restriction effect increases the cost of the spatially aggregated patch arrangements? The reason is the presence of a third effect that has not been considered yet: a surplus transfer effect. The agglomeration payment not only aggregates patches in space but also reduces the producer surplus of the land-owners. Without a density threshold $\left(\rho_{\min }=0\right)$, only land-owners with positive individual profits, $\pi_{i}>0$, participate. If a positive density threshold is set and only land-owners with positive individual profits participate, their number may be too small to reach the density threshold $\rho_{\text {min }}>0$ and so they may not get any payments at all (Eq. 2). To get at least some profit individual land-owners have to sacrifice some of their producer surplus and transfer it through side-payments (Eq. 3) to the land-owners whose individual profits would otherwise be negative. Now the density threshold is reached and a positive total profit obtained. To give a numerical example, set $p=2.6$ instead of $p=3.1$ in Fig. 2. The profit maximising patch configuration can be achieved only if the owners of the two $(c=2)$ patches offer a side payment to the owner of the $(c=3)$-patch. Both the required budget (7.8) and the total profit $(7.8-7=0.8)$ are reduced compared to the case of $p=3.1$.

The efficiency gain of the agglomeration payment depends on the budget. First we note that connectivity monotonously increases with increasing budget. The reason is that an increasing budget allows offering higher payments. It becomes profitable for the landowners then to expand the rectangle and manage more patches green, which increases connectivity $\Gamma$. However, the increase of $\Gamma$ with increasing budget is non-linear and depends on the density threshold $\rho_{\min }$. Figure 2a shows that high efficiency gains exist only 
if the budget is below a specific value. For instance, in the case of $\rho_{\min }=0.6$ the connectivity (long-dashed line) exceeds the connectivity obtained from homogenous payments (solid line) only for budgets below $80 m_{c}$, while for higher budgets both curves are identical, indicating zero efficiency gain. The reason is that at high budgets there are already many green patches in the landscape and selecting one patch instead of another will not increase connectivity considerably (the connectivity effect is small). ${ }^{1}$ Furthermore, in such a situation the density threshold can be reached in the entire landscape already with those patches that lead to any non-zero density. In this case, there is no incentive for the owners of these patches to share their producer surplus (the surplus transfer effect is small). Each of them gains his full amount $p-c_{i}$, which is just what one obtains under homogenous payments. Overall, the highest efficiency gain in relative terms occurs with small budgets and decreases with increasing budget.

Comparison of Figs. (2a) and (2b) reveals that for species with long-range dispersal efficiency gains are smaller than for species with short-range dispersal. This is plausible given that good dispersers are less dependent on the spatial configuration of habitat than poor dispersers (e.g., Drechsler et al. 2007), which means that for good dispersers the connectivity effect is smaller than for poor dispersers.

The influence of the cost variation $(\sigma)$ is ambiguous (compare Figs. (2a) and (2c). For small budgets higher variation in the costs $c_{i}$ slightly reduces the cost-effectiveness of the agglomeration payment while for high budgets it increases the cost-effectiveness of the agglomeration payment. The reason for the ambiguity is that for small budgets the patch restriction effect is relatively strong, because a small budget means that patches that in the case of homogenous payments are dispersed over the entire landscape have to be in a very small rectangle, which leads to a high likelihood that costly patches have to be selected. For increasing budgets the rectangle becomes larger and the patch restriction effect decreases to zero (reached when the rectangle covers the entire landscape). The surplus transfer effect, in contrast, increases with the budget and a point exists where both effects have the same magnitude, so that for relatively small (large) budgets the patch restriction (surplus transfer) effect dominates and increases (decreases) the costs of the agglomeration payment compared to homogenous payments.

\section{SUMMARY AND DISCUSSION}

Designing effective compensation payment schemes for biodiversity conservation requires accounting for the spatial configuration of habitat patches. While ecological benefits increase with increasing spatial connectivity of patches the costs are expected to increase, too, as more costly patches tend to be selected. This raises the open question of how costeffective an agglomeration payment is relative to spatially homogeneous payments. Herein we address this question at both a conceptual level and for a specific conservation study the design of payments for butterfly-friendly grassland management in the region of Landau, Germany. We find the cost-effectiveness of an agglomeration payment is determined by the interaction of three mechanisms: (i) the connectivity effect, (ii) the patch restriction effect, and (iii) the surplus transfer effect.

The connectivity effect (i) arises because ceteris paribus a spatially aggregated arrangement of habitats usually leads to higher survival rates of endangered species than spatially dispersed habitats and the agglomeration bonus increases the ecological effectiveness of the habitat network. The connectivity effect is strongest when the conservation budget or the dispersal range of the species is small, or both. The reason is that in the case of a small budget, only few habitats are created which under homogenous payments are dispersed all over the landscape. The distances between these patches are too large for the species to cross, especially if the species has a small dispersal range. Spatial aggregation of these few patches strongly improves the dispersal among them. The dispersal range of the Large Blue

\footnotetext{
${ }^{1}$ This result is similar to Lewis and Plantinga (2007) who also find that the percentage of the landscape covered with land use beneficial to conservation has a significant impact on the relative performances of different conservation policies.
} 
butterfly analysed in the case study is so small (on average ca. $1 \mathrm{~km}$ ) that for homogeneous payments and small budgets the distances between habitat patches are often larger than the species' dispersal distances, and so the connectivity effect is relevant.

The patch restriction effect (ii) arises, because different patches are selected under homogeneous payments and under the agglomeration payment. Under homogeneous payments the most inexpensive patches out of all patches in the landscape are chosen. In contrast, under the agglomeration payment selection is restricted and can be made only from the patches contained in a smaller compartment (rectangle $R$ ) of the landscape. As a consequence, selecting $N$ patches from the compartment is more expensive than selecting the same number of patches out of the entire landscape. This cost increase, or the patch restriction effect, is largest when the cost heterogeneity $(\sigma)$ among the patches in the landscape is high.

The surplus transfer effect (iii) is a consequence of the density threshold $\rho_{\min }$ that has to be exceeded by the land-owners to receive a payment. Depending on the specific situation, some of the land-owners will be confronted with the choice between not receiving any payment and offering side-payments to farmers whose participation in the programme is necessary to reach the density threshold but whose individual profits would be negative without the side payments. The side-payments reduce the overall producer surplus - an effect that is largest when the cost heterogeneity $(\sigma)$ among the patches in the landscape is high.

The surplus transfer effect is responsible for our unexpected result that the efficiency gain of the agglomeration payment is always positive (Fig. 2). Without the surplus transfer effect there would be the expected trade-off between connectivity and patch restriction effect, and too high cost variation would cause the patch restriction effect to dominate the connectivity effect, leading to negative efficiency gains.

Our result that the agglomeration payment leads to a positive efficiency gain is unambiguous within the parameter range considered. The open question is whether different parameters would make things worse than the status quo, i.e., negative efficiency gains. Efficiency might be negative if the species dispersal range is vast. This implies the connectivity effect is close to zero and, as a consequence, the patch restriction effect may dominate the surplus transfer effect. The agglomeration payment, however, is not designed for such situations. Rather the purpose of the bonus is to provide incentives to generate a certain spatial arrangement of habitats which is unnecessary for vast dispersal ranges.

Efficiency gains may also become negative if costs are spatially correlated. In our study we assumed the costs are uncorrelated among patches. But with spatial cost correlations the implied clustering of low-cost patches in space may induce ecologically sufficient clustering of habitats even with homogenous payments. An agglomeration payment would not improve the ecological benefit and the additional costs associated with the patch restriction effect could render the agglomeration payment less cost-effective than homogeneous payments. A detailed analysis of the effects of spatial correlation is worthy direction for future research.

A possible alternative to the agglomeration bonus may be that the regulator negotiates individually with those land owners whose land would be needed for a certain spatial configuration. This, however, requires that the regulator has a high level of information about each individual land owner's conservation costs which seems unrealistic (cf. Lewis and Plantinga [2007]). In contrast, for the agglomeration payment the regulator only needs to know mean and variance of the costs. The negotiation partners are neighbouring land owners and conditions do arise in which each landowner might know more about his or her neighbour than the regulator. In local rural agricultural communities many producers are adjacent to each other, know each others preferences and have a vested interest in knowing what their neighbours are doing - e.g. in pest control decisions.

For the agglomeration payment to work, however, land owners need to coordinate with each other about side payments and their actions. This implies additional transaction costs 
arise relative to standard homogeneous payments. These transaction costs might reverse our result that agglomeration payments are more cost-effective than homogeneous payments. To what extent these transactions costs arise is an empirical matter and depends on factors such as the local tradition of cooperation between land owners, the information disparity across land owners about conservation costs and the number of land owners that need to participate in negotiations. Our analysis indicates this number is likely to be small because efficiency gains are highest in relative terms for small conservation budgets. A small budget implies only a few land owners need to participate in negotiations, which makes negotiation less costly than for a large number of land owners.

We appreciate the limits of our results - the agglomeration bonus is less likely to be successful the greater the information disparity across neighbouring producers, and the more severe the problems of cooperation. Assessing the robustness of the agglomeration bonus idea based on the determinants and degrees of shared knowledge and cooperation across neighbouring producers remains an important area for future empirical research. Nevertheless, bearing in mind that in Europe and the US several billion Euro and dollars are spent each year on conservation our result suggests that significant improvements in conservation management could be made if agglomeration payments are used in practise.

\section{REFERENCES}

Bergmann, H., Berechnung von Kosten für Maßnahmen zum Schutz von gefährdeten Maculinea-Arten. UFZ discussion paper 2/2004, UFZ Leipzig-Halle GmbH (http://www.ufz.de/data/ufz-diskussionspapier2-20041180.pdf), 2004.

Brown, G.M., Jr., and J.F. Shogren, Economics of the Endangered Species Act, The Journal of Economic Perspectives 12(3), 3-20, 1998.

Drechsler, M., F. Wätzold, K. Johst, H. Bergmann, and J. Settele, A model-based approach for designing cost-effective compensation payments for conservation of endangered species in real landscapes, Biological Conservation, 140(174) -186, 2007.

European Commission, Agri-environment Measures: Overview on General Principles, Types of Measures, and Application. Study of the European Commission Directorate General for Agriculture and Rural Development, Unit G-4 - Evaluation of Measures applied to Agriculture (URL: http://europa.eu.int/comm/agriculture/publi/reports/agrienv/rep_en.pdf), 2005.

Frank, K., and C. Wissel, A formula for the mean lifetime of metapopulations in heterogeneous landscapes, American Naturalist 159, 530-552.

Hanski, I., Metapopulation Ecology, Oxford University Press, Oxford, UK, 1999.

Johst, K., M. Drechsler, J. Thomas, J., and J. Settele Influence of mowing on the persistence of two endangered large blue butterfly species, Journal of Applied Ecology 43, 333-342, 2006.

Knight R.L, and P.B. Landres (Eds.), Stewardship across boundaries, Island Press, Washington, DC, USA, 1998.

Landell-Mills, N., and I. Porras, Silver Bullets or Fools' Gold? A Global Review of Markets for Forest Environmental services and Their Impact on the Poor, International Institute for Environment and Development (IIED), London, UK, 2002.

Lewis, D.J., and A.J. Plantinga, Policies for habitat fragmentation: combining econometrics with GIS-based landscape simulations. Land Economics 83, 109-127, 2007.

Parkhurst, G.M., J.F. Shogren, P. Bastian, J. Kivi, J. Donner, and R.B.W. Smith, Agglomeration bonus: an incentive mechanism to reunite fragmented habitat for biodiversity conservation, Ecological Economics 41, 305-328, 2002.

Parkhurst, G., and J. Shogren, Spatial incentives to coordinate contiguous habitat, Ecological Economics 64, 344-355, 2007.

Parkhurst, G., and J. Shogren, Smart subsidies for conservation, American Journal of Agricultural Economics 90, 1192-1200, 2008.

Raymond, L., Cooperation without Trust: Overcoming collective action barriers to endangered species protection, Policy Studies Journal 34, 37-57, 2006.

Wätzold, F., N. Lienhoop, M. Drechsler, and J. Settele, Estimating optimal conservation in agricultural landscapes when costs and benefits of conservation measures are heterogeneous in space and over time, Ecological Economics 68, 295-305, 2008. 